\title{
Zero-IF and Modularization RF Design of Wireless Identification Reader for UHF Passive Tags
}

\author{
Linsen Li, Gongliang Chen, Yue Wu, Jianhua Li \\ School of Electronic, Information and Electrical Engineering \\ Shanghai Jiao Tong University \\ Shanghai, China \\ 1sli@sjtu.edu.cn
}

\begin{abstract}
In this paper, a zero-IF and modularization RF design scheme of wireless identification reader for UHF passive tags is presented. The reader works at $860-960 \mathrm{MHz}$, and corresponds to the standard of EPC C1 G2 and ISO/IEC 18000-6C. The realization of some key modules is elaborated. The tests have shown that the proposed RF design scheme of reader works well. The transmit power of the reader is up to $32 \mathrm{dBm}$, and the stable reading range reaches 9 meters.
\end{abstract}

Keywords-wireless identification; reader; UHF passive tag; zero-IF; modularization

\section{INTRODUCTION}

High performance and low cost has changed UHF passive technology into the application mainstream of Internet of Things based on wireless identification, or RFID (RFID IoT). However, in the practical application, it is well known that one of the bottleneck problems in RFID IoT is the design of reader, because one reader must manage at least thousands of tags. Therefore, high-quality wireless identification reader (WIR) for UHF passive tags stands for one of the core techniques in the industrialization of UHF RFID.

Some of design schemes of UHF RFID readers have been proposed [1-10]. But these schemes either stayed in simulation level, or were based on the existing single RF chips. In other words, they didn't focus on the practical challenges, or were not so flexible in realization.

In this paper, we propose a zero-IF and modularization RF design scheme of WIR for UHF passive tags based on discrete elements and small scale ICs. The reader works at $860-960 \mathrm{MHz}$, and corresponds to the standard of EPC C1 G2 and ISO/IEC 18000-6C. The scheme is simple and flexible, and will be changed into real product.

\section{PROPOSED RF DESIGN SCHEME} Fig. 1,

Our proposed RF design scheme is shown in Fig. 1. In

Crystal Oscillator is used to generate the reference frequency of PLL (Phase-Lock Loop). Here, the working frequency is $33 \mathrm{MHz}$.

PLL is used to multiply the $33 \mathrm{MHz}$ to the demand frequency of EPC C1 G2, that is, $915 \mathrm{MHz}$.
Modulator is used to modulate the working frequency to generate the modulated RF signal.

Power Amplifier (PA) is used to magnify power to the demand scope of the EPC C1 G2, for example, the maximum value is $32 \mathrm{dBm}$.

Circulator is used for common use of the transmitter and the receiver, i.e., to transmit modulated signal and to receive the return signal.

The function of antenna is to transmit and receive the EM wave.

The function of demodulator is to demodulate the return signal.

The function of differential amplifier is to magnify the demodulated signal. It can enhance the receiving sensitivity.

The function of comparator is to reshape the waveform of the demodulated and magnified signal for the processing convenience of the controller.

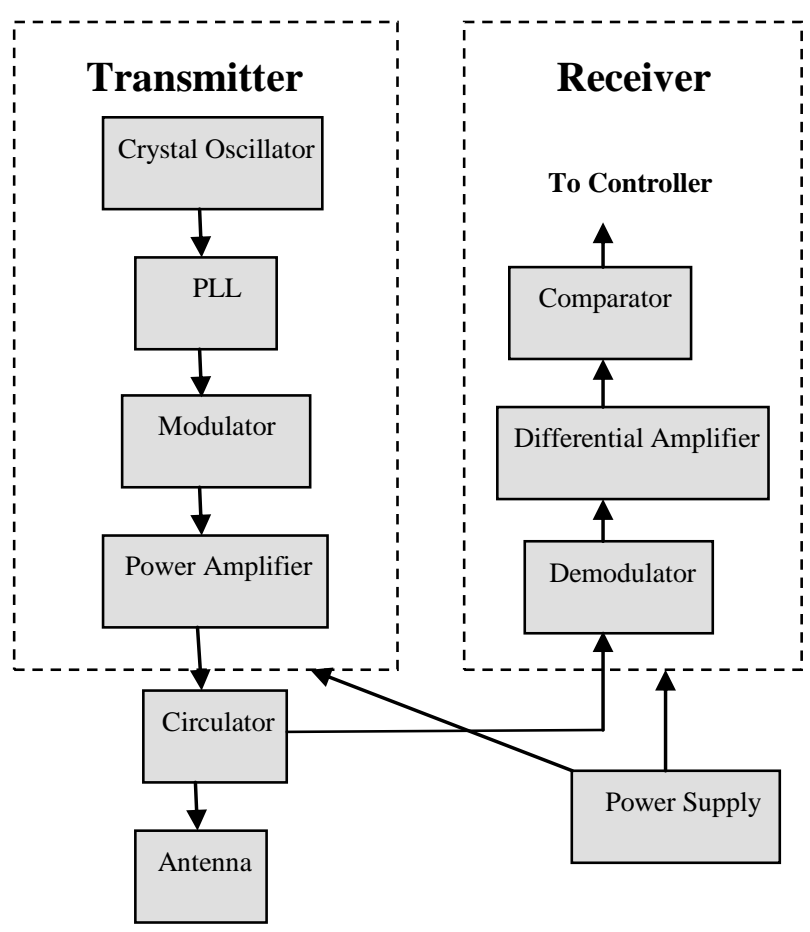

Fig. 1 Proposed RF design scheme of WIR 


\section{Realization of Some Key Modules}

A. Crystal Oscillator

An active crystal oscillator chip named F33.000 from JVC is adopted here. It works at $33 \mathrm{MHz}$. The concrete realization of Crystal Oscillator is shown in Fig. 2.

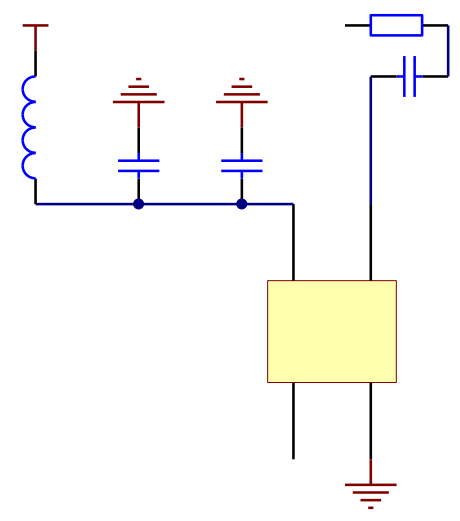

Fig. 2 Realization of Crystal Oscillator

\section{$B$.}

$$
P L L
$$

The core chip is PLL400-915 whose working frequency is from $902 \mathrm{MHz}$ to $928 \mathrm{MHz}$, and its center frequency is $915 \mathrm{MHz}$. Its typical output phase noise is $-111 \mathrm{dBc} / \mathrm{Hz}$, and the typical second harmonic suppression is $-15 \mathrm{dBc}$. The concrete realization of PLL is shown in Fig. 3.

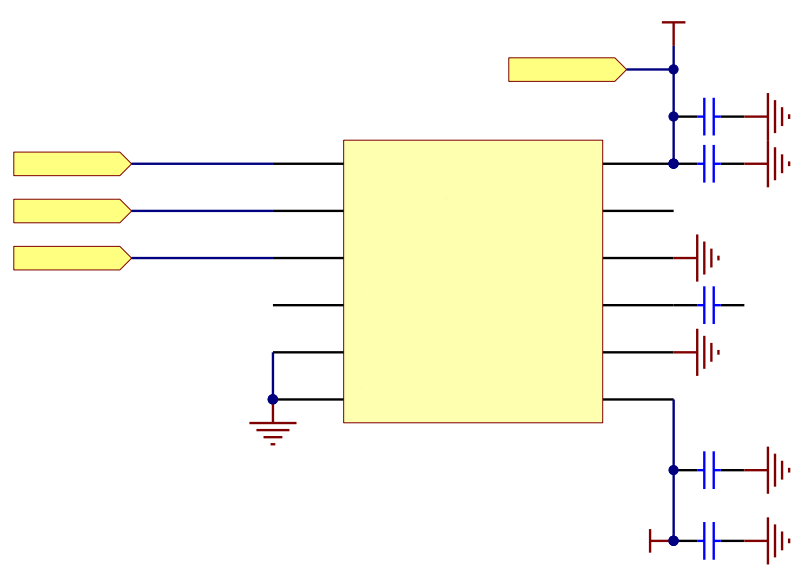

Fig. 3 Realization of PLL

\section{C.}

Modulator

The chip of HMC194 is utilized here. It is a GaAs MMIC SPDT switch, and can control signals from DC to $3 \mathrm{GHz}$. It has been optimized to provide $50 \mathrm{~dB}$ high isolation between two RF paths with minimal insertion loss of $0.7 \mathrm{~dB}$. The concrete realization of Modulator is shown in Fig. 4.

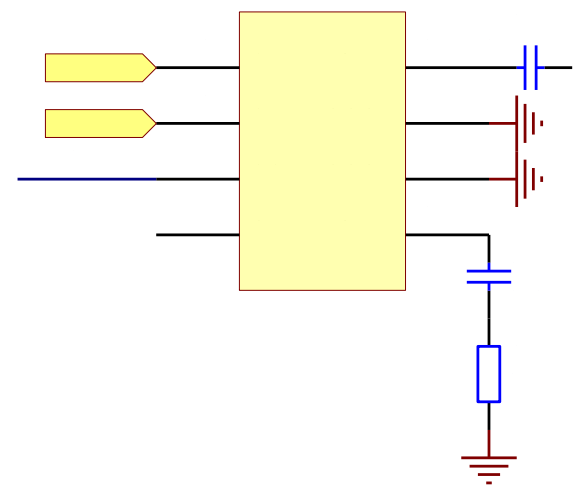

Fig. 4 Realization of Modulator

\section{Power Amplifier}

PF01411A is the core chip. The chip is a MOS FET power amplifier module, and a high gain 3 stage amplifier with $0 \mathrm{dBm}$ input. It owns wide gain control range of $90 \mathrm{~dB}$. The output power is $4.3 \mathrm{~W}$, and can be adjusted by pin $\mathrm{V}_{\mathrm{APC}}$. This is very important to change the reading range of WIR. The concrete realization of PA is shown in Fig. 5.

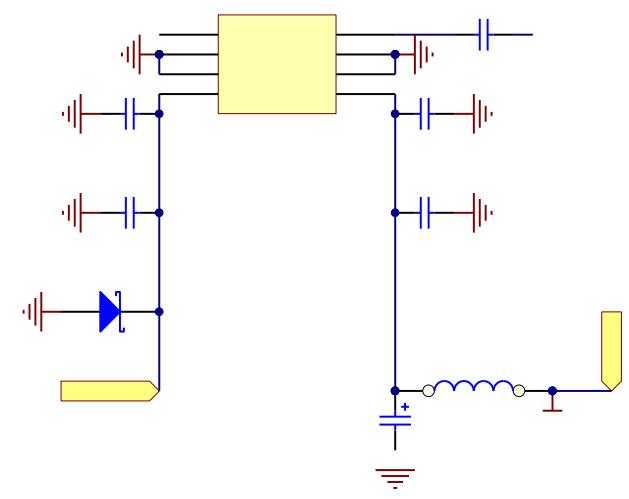

Fig. 5 Realization of PA

\section{TEST AND ANALYSIS}

The scheme described in II and III has been implemented by 4-layer FR-4 PCB. The size of the PCB is $160 \mathrm{~mm} \times$ $100 \mathrm{~mm}$. The picture of real RF product of WIR is shown in Fig. 6.

In Fig. 6, the upper part is the Transmitter, the lower part is the Receiver. In the upper part, from left to right, you can find the Crystal Oscillator, PLL, Modulator, PA, Circulator and the interface to the reader Antenna. In the lower part, from right to left, you can find the Demodulator, Differential Amplifier, and Comparator. 


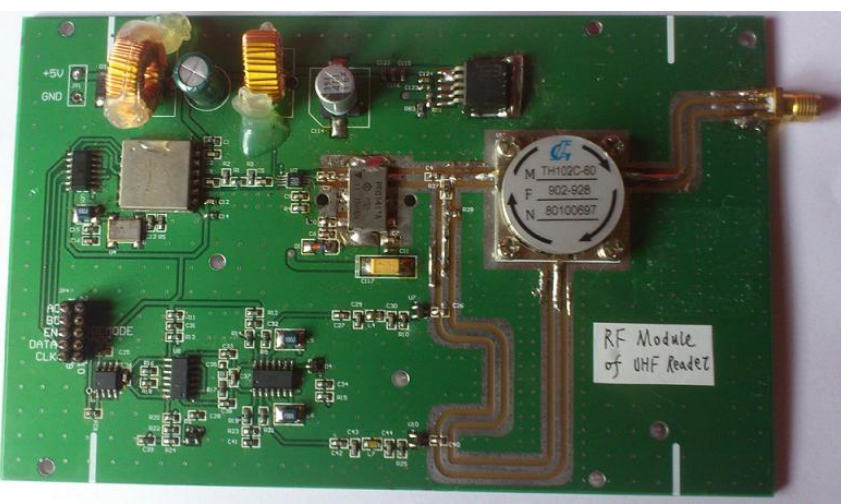

Fig. 6 Picture of real RF product of WIR

The waveforms of real RF product of WIR in Fig. 6 using oscilloscope are shown in Fig. 7. These two waveforms illustrate the VCC of PA (shown in yellow color) and the output of Circulator (shown in green color). They indicate that the working voltage of the PA is $4 \mathrm{~V}$, and that the output of Circulator (to the interface of the reader antenna) is the expected modulated signal. At the same time, the two waveforms have good synchronization performance.

The transmit power of the reader is up to $32 \mathrm{dBm}$, and the stable reading range reaches 9 meters.

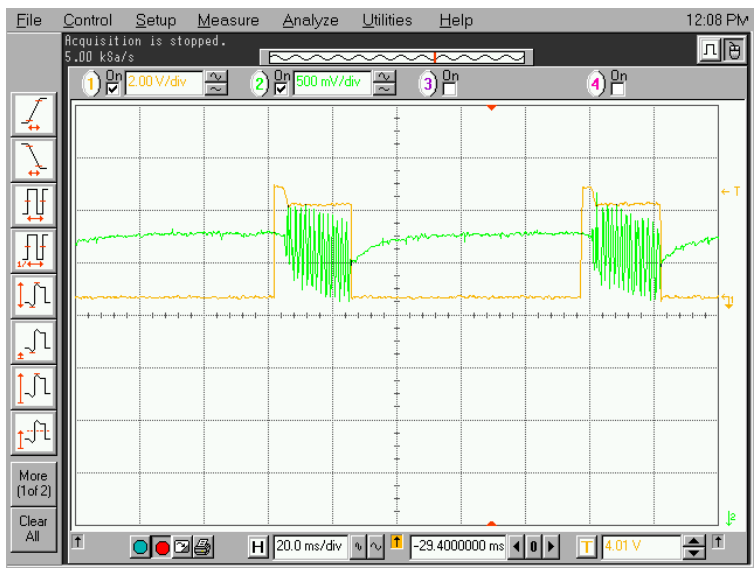

Fig. 6 Waveforms of 1) VCC of PA (shown in yellow color) 2) Output of Circulator (shown in green color)

\section{CONCLUSION}

In this paper, we have presented a zero-IF and modularization RF design scheme of WIR for UHF passive tags. The reader can works at $860-960 \mathrm{MHz}$, and corresponds to the standard of EPC C1 G2 and ISO/IEC 18000-6C. We have elaborated the realization of some key modules. The test have shown that the proposed RF module of reader work well. The transmit power of the reader is up to $32 \mathrm{dBm}$, and the stable reading range reaches 9 meters.

\section{ACKNOWLEDGMENT}

This work was supported by the National Natural Science Foundation of China under Grant No. 61071078, the Doctoral Foundation of Priority Development Areas of Ministry of Education under Grant No. 20130073130006, and the Opening Project of Key Lab of Information Network Security of Ministry of Public Security.

\section{REFERENCES}

[1] A. Marzukil and S. David, Design of $920 \mathrm{MHz}, 0.8 \mathrm{um}$ CMOS Low Noise Amplifier for UHF RFID Reader, 2006 INTERNATIONAL RF AND MICROWAVE CONFERENCE PROCEEDINGS, SEPTEMBER 12 - 14, 2006, PUTRAJAYA, MALAYSIA, pp.149151.

[2] Sang Kyu Kim, Sung Sik Nam, and Sung Ho Cho, A FPGA DESIGN OF THE I/Q SIGNAL COMBINING FOR UHF RFID READER RECEIVER, Proceedings of IC-NIDC 2010, pp.556-560.

[3] Povalac, A., Zamazal, M., and Sebesta, J., Firmware Design for Multi-Protocol UHF RFID Reader, 2010 20th International Conference Radioelektronika, pp. 1-4.

[4] Mayordomo Iker, Ubarretxena Aritz, Valderas Daniel, Berenguer Roc, and Gutierrez Inigo, Design and Analysis of a Complete RFID System in the UHF Band Focused on the Backscattering Communication and Reader Architecture, 2007 3rd European Workshop on RFID Systems and Technologies (RFID SysTech), 2007 , pp. 1-6.

[5] Jin-Min Song, Guang-Bin Wang, and Bi-Cheng Xiao, Design of UHF RFID Reader Based on Embedded Linux, 2012 Fourth International Conference on Computational and Information Sciences, pp. 10011004

[6] Zhao Lei, Huang Jiong, Wang Wen ping, and Mei Xiao han, The Design Of Zero-IF Receiver Of UHF RFID Reader, 2011 Cross Strait Quad-Regional Radio Science and Wireless Technology Conference, pp. 1058-1061

[7] Michael Buettner, and David Wetherall, A Software Radio-based UHF RFID Reader for PHY/MAC Experimentation, 2011 IEEE International Conference on RFID, pp. 134-141.

[8] Taehwan Joo, Hongtak Lee, Sunbo Shim, and Songcheol Hong, CMOS RF Power Amplifier for UHF Stationary RFID Reader, IEEE MICROWAVE AND WIRELESS COMPONENTS LETTERS, VOL. 20, NO. 2, FEBRUARY 2010, pp. 106-108.

[9] Min-Su Kim, Sung-Chan Jung, Jonghyuk Jeong, Hyungchul Kim, Mincheol Seo, Junghyun Ham, Cheon-Seok Park, and Youngoo Yang, Adaptive TX Leakage Canceler for the UHF RFID Reader Front End Using a Direct Leaky Coupling Method, IEEE TRANSACTIONS ON INDUSTRIAL ELECTRONICS, VOL. 61, NO. 4, APRIL 2014 pp. 2081-2087.

[10] Lu Liang, Zhang Chun, Wang Jing Chao, A Digital IF Based UHF RFID Reader Transmitter, 2010 IEEE Asia Pacific Conference on Circuits and Systems (APCCAS), 2010, pp. 96-966. 